\title{
Why Traditional Usability Criteria fall short in Ambient Assisted Living Environments
}

\author{
Martina Ziefle \\ Communication Science, \\ Human-Computer Interaction Center \\ RWTH Aachen University, \\ Campus-Boulevard 57 \\ 52074 Aachen, Germany \\ ziefle@comm.rwth-aachen.de
}

\author{
Wiktoria Wilkowska \\ Communication Science, \\ Human-Computer Interaction Center \\ RWTH Aachen University, \\ Campus-Boulevard 57 \\ 52074 Aachen, Germany \\ wilkowska@comm.rwth-aachen.de
}

\begin{abstract}
According to ISO 9241, traditional usability definitions focus on pragmatic aspects of technical systems, such as the ease of using a system (effectiveness, efficiency) and the perceived usefulness. While this definition was found to be highly applicable for a wide range of technical systems in the working context, it is a major question if the pragmatic approach is adequate for novel technical developments (e.g., health supporting systems) in the home sector. The integration of medical technology in home environments, such as Ambient Assisted Living (AAL) requests a high willingness of users to accept new forms of medical assistance and to rely on the technology in a very sensitive and intimate field. Also, user diversity is a serious issue, as increasingly more, and especially older users will have to master technical devices in the near future. In order to contribute to a basic acceptance understanding of such medical technologies against the background of user diversity, in this research, along with the traditional usability criteria, additional aspects of system usability are examined. Results show that the sole consideration of ease of use and the perceived usefulness is not sufficient for current designs. Future usability concepts should much more integrate additional aspects like trust in technology, wish for privacy protection, system reliability and fun, especially when designing technology solutions for older users.
\end{abstract}

\section{Keywords}

Ambient Assisted Living (AAL), older adults, user diversity, usability, technology acceptance, medical technology

\section{INTRODUCTION}

Innovations in the field of medical technology like the integration of sensors and health monitoring systems into home environments are potential approaches to solve the gap arising from the decreasing availability of care persons and the increasing needs of medical care for older adults [9]. The so-called telemedical or eHealth applications within AAL environments refer to a

Permission to make digital or hard copies of all or part of this work for personal or classroom use is granted without fee provided that copies are not made or distributed for profit or commercial advantage and that copies bear this notice and the full citation on the first page. To copy otherwise, to republish, to post on servers or to redistribute to lists, requires prior specific permission and/or a fee.

User Centered Design 2014, May 20-22

Copyright (C) 2014 ICST 978-1-63190-011-2

DOI 10.4108/icst.pervasivehealth.2014.255481 combined use of information and communication technologies (ICT) and health monitoring devices [11].Electronic health applications (eHealth) have the potential to reduce costs, provide healthcare services remotely, and increase the efficiency of such services. Hence, an effective integration of eHealth applications could improve peoples' quality of life by enabling safer independent living and increased social inclusion. In addition, electronic healthcare opens up new possibilities for supporting medical diagnosis and therapy by bridging temporal and spatial gaps between patients and physicians. ICT enables an autonomous and unobtrusive collection of clinical data, and supports the continuous transmission of physiological information between patients and remote healthcare providers [12]. For patients especially the older ones - such systems allow a safe healthcare and an independent living in the own home environment.

While the maturity of technical and medical systems is far reaching, ready to be implemented into home environments, there is still a broad reluctance of persons to accept such novel developments in their private homes [13]. Although the vital importance of ensuring that the technology produced is both usable and appropriate for diverse user groups, recognition of the importance of diversity and the integration of users' needs is only slowly influencing mainstream usability studies [17]. Another reason for this public reluctance is the uncertainty of users, if and to which extent the novel developments do actually respect individual values, such as need for privacy and data security [14]. Design approaches have thus to undergo a profound change. This includes that the mere focus on the traditional usability concept might be extended, focusing not only on pragmatic aspects of technology use, but also, on individual concerns such as trust in technology, data security, privacy, and not least, issues of design and the emotional value of technical systems for their users [6].

\subsection{Usability and Technology Acceptance}

The majority of approaches dealing with technology acceptance refer to the acceptance of ICT in a job-related context. According to such models (e.g., TAM [4]), technology acceptance is exclusively influenced by the perceived ease of using a system and the perceived usefulness. Yet, only few studies concentrated on the diversity of users and their acceptance patterns [1] [5] even though it is obvious from daily life experience that people may have different adoption behaviors due to individual factors. Another critical issue of existing technology acceptance models is that approaches exclusively focus on acceptance patterns of ICT in a working context and it is highly disputable, if those concepts might be also applicable for the integration of medical technology in the home context, as it is the case in AAL systems [8]. Beyond its importance for patients' safety, medical technology refers to 
"taboo-related" areas, which are associated with disease and illness [17]. In addition, medical monitoring technology is often perceived as stigmatizing and breaking into persons' intimacy and privacy spheres, which may lead to the feeling of being permanently controlled [14], especially in older and frail persons. The exclusive concentration on functional components can be also found in the traditional usability concept. According to ISO 9241, the pragmatic aspects of technology, covered by the term "usability", are measured by effectiveness (how successful is the interaction), efficiency (how fast is the interaction), and satisfaction (how satisfied are users during the interaction). Though, considering the complexity of (future) health supporting technology as well as the increasing diversity of users, contexts, and technology types, the concentration on pragmatic aspects is not sufficient. Traditional approaches usually do not reflect the importance of (positive) emotions or concerns. Facing the novel technology environments, a broadening of the focus is required and an inclusion of emotional or affective aspects into designs is needed [15]. Thus, usability should be described as a complex out of pragmatic aspects that include attributes emphasizing individuals' productivity, but at the same time, it should take the affective and hedonic aspects, as well as the attributes emphasizing individuals' wellbeing, pleasure and fun when interacting with technology into account. Moreover, emotional facets such as trust, privacy and aspects of security should be equally considered.

\section{METHOD}

In order to understand, how different usability dimensions of a medical system integrated into natural home context is evaluated, we used an experimental Living Lab environment. In the first step participants interacted with medical technology integrated into an experimental living room, Future Care $\mathrm{Lab}^{\mathcal{O}}$ (Figure 1), and evaluated then the usability in a comprehensive way.

\subsection{The Future Care Lab ${ }^{\circledR}$}

As experimental environment, the Future $\mathrm{Care}^{\mathrm{Lab}}{ }^{\odot}$ at RWTH Aachen University was used (Figure 1). The lab is technically realized as an intelligent living room, equipped with different medical assist devices and interaction interfaces. Its purpose is not only to test novel, integrative prototypes for personal healthcare systems in future home environments, but also to serve as a test bed for user studies. A full-scale prototype room a had been built up enabling researchers to test experimental interfaces with test persons of different ages and health states.

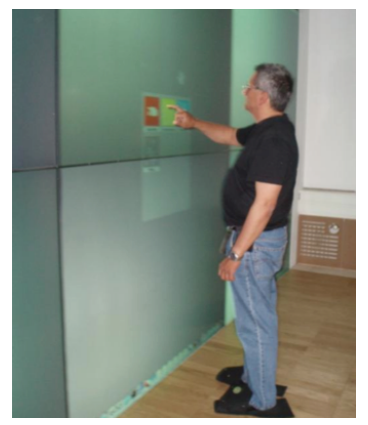

Figure 1: Participant during the interaction with the smart wall in the Future Care Lab $^{\odot}$ [7].

Different parts of the room (walls, floor, furniture) are used as input and output modalities for medical services, but also for daily

\footnotetext{
${ }^{1} \mathrm{http}: / /$ www.comm.rwth-aachen.de/index.php?article_id=355\&clang=0
}

activities. The (smart) wall of the living room represents a huge multi touch display covering a plane of $4.8 \mathrm{~m} \times 2.4 \mathrm{~m}$. Different interaction modes are experimentally evaluated, comparing direct and indirect mechanisms.

\subsection{Participants}

A total of 13 individuals participated in the survey $(53.8 \%$ female). Their age ranged from 19-59 years $(\mathrm{M}=32.1, \mathrm{SD}=11.6)$. Participants were reached through the social networks of younger and middle-aged adults. They were not remunerated for their efforts, but were keen to learn about innovations in ambient AAL.

\subsection{The Questionnaire Instrument}

In order to extend the traditional usability concept, a questionnaire instrument was developed that included more aspects of usability, which might be important from the users' point of view (see Table 1). The items used were based on previous empirical work, in which we collected argumentation patterns and user experience (focus groups) of men and woman of a wide age range. The first part assessed demographic characteristics. In the second part participants evaluated the usability of the system according to different dimensions. Each question was answered on a 7-point Likert Scale ranging form 1 (='strongly disagree') to 7 (='strongly agree'). For analyzing purposes, total scores per dimension were formed. Negatively formulated items were reversed.

Table 1. Items and dimensions of the questionnaire

Ease of Use / Complexity of the system

"I had no difficulties using this system."

"I thought the system was unnecessarily complex."

"I had to learn a lot of details before I could use the system."

"The use of this system would ease my everyday life."

\section{Perceived Usefulness}

"I consider this system useful."

"I do not think this technology will support me in my daily routine."

"I can benefit from this system in many respects."

"I cannot see the use of this system."

Comprehensibility

"The functions and menu structure of the system were comprehensible."

"To me, the system was not fully developed and hard to understand."

"I had difficulties learning to operate the system."

"I can imagine that most people will learn the handling of the system rather quickly."

Fun / Motivation

"I enjoyed using the system."

"I like the system's design."

"I am generally not fond of using such systems."

Functionality

"To me, the different functions of the system are well integrated."

"To me, the functions of the systems are clear and comprehensible."

"It was easy for me to take care of things with the help of the system."

"The menu display corresponds to the actual functions."

Intention to use

"I doubt I would need a system like this in my every day life."

Trust

"I deem this system trustworthy."

"I do not trust this system enough to rely on it completely."

Privacy
"It bothers me that my data might be accessible by others."
"I wish for a personal access code for the system to protect my privacy."




\begin{tabular}{l}
\hline Data security \\
\hline "I felt safe while using the system." \\
"I do believe that the data security is ensured." \\
"I do not regard the system to be sufficiently reliable." \\
\hline Governance \\
"A regular check of my physical data and vital signs would give me the \\
uncomfortable feeling of being under constant surveillance." \\
"I do think it sensible to have the system regularly check my physical \\
data and vital signs (e.g., blood pressure, weight)." \\
"I want to be in charge of the system at all times, be it via access codes, \\
passwords, or the like."
\end{tabular}

\subsection{Procedure}

In the first step, participants were introduced into the topic of AAL and the potential of telemedicine. Next, the experimenter demonstrated how to interact with the system (measures of weight and blood pressure). Participants' task was to take the same measures of the mentioned vital signs, navigating through the system menu structure. After collection of these data, participants navigated to the graphical measurement overview to be able to judge the benefit of the system's functionality. The test persons were informed that in a real-life scenario the vital data would be stored in an online database. After completing the interaction with the system, participants finally filled in the usability questionnaire. Participants were tested individually, and the duration of each test unit did not exceed 30 minutes.

\section{RESULTS}

All participants successfully completed the task of interacting with the system to measure their weight and blood pressure, and reached thus a high effectiveness. This result shows an overall high functional system quality. In the following section we report on the evaluation of the different usability dimensions. In a first step, evaluations of the single aspects were analyzed descriptively. As the different dimensions consisted of a varying number of items, the scores were normalized (\%). In the next step, Spearman correlations were conducted in order to understand how the different dimensions of the extended usability measurement relate to each other. The level of significance was set at $5 \%$.

\subsection{Evaluation of the System Usability}

The overall evaluations are depicted contrasting the dimension scores of the younger $(<29, \mathrm{~N}=6)$ and the older user group $(>29$, $\mathrm{N}=7$ ). Figure 2 shows the results.

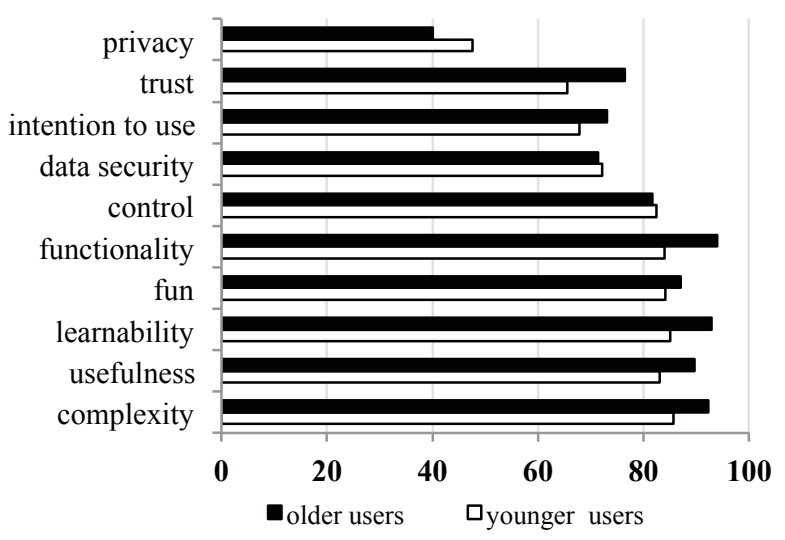

Figure 2: Overall evaluation scores (in \%) in the younger (white bars) and the older user group (black bars).
As presented in Figure 2, evaluations reach quite high scores (complexity, usefulness, learnability, fun, functionality). It is noteworthy, however, that evaluations are more positive in the older in contrast to the younger group. Striking result was found for privacy, which was evaluated as quite low in the setting.

\subsection{Interrelation of Usability Dimensions}

In order to understand the impact of user diversity, correlation analyses were run for age groups separately. As found, highly significant correlations between the different usability dimensions were revealed for both age groups, though completely different in nature and direction. In Figure 3, correlations for the younger group are visualized, in Figure 4 for the older group.

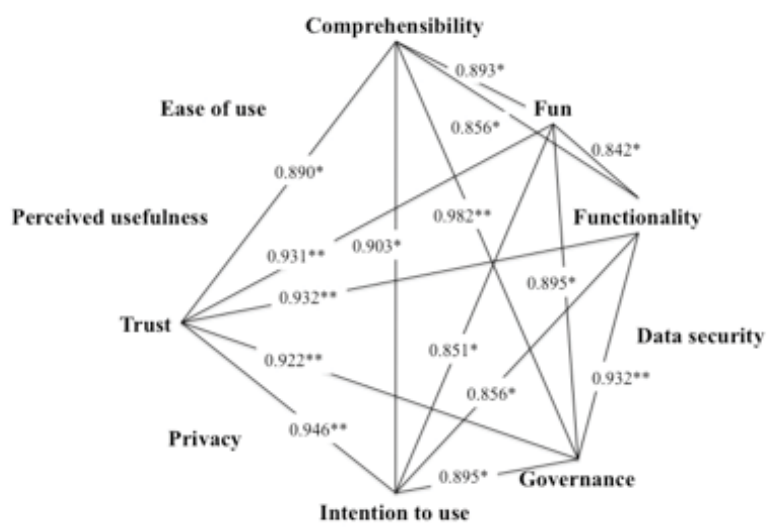

Figure 3. Interrelation between usability dimensions for the younger user group $(* p<0.05 ; * * \mathbf{p}<0.01)$

Considering the correlation outcomes in the younger group (Figure 3), it becomes evident that the classical criteria of usability - ease of use and usefulness - are neither significantly related to each other nor to the other aspects under study. Taken from the high correlation scores, trust, comprehensibility, functionality, governance, and fun are important aspects for participants and the intention to use such systems. Characteristically, the nature of the relation is positive (e.g. the higher the comprehensibility, the higher the trust). The picture is completely different in the older group (Figure 4).

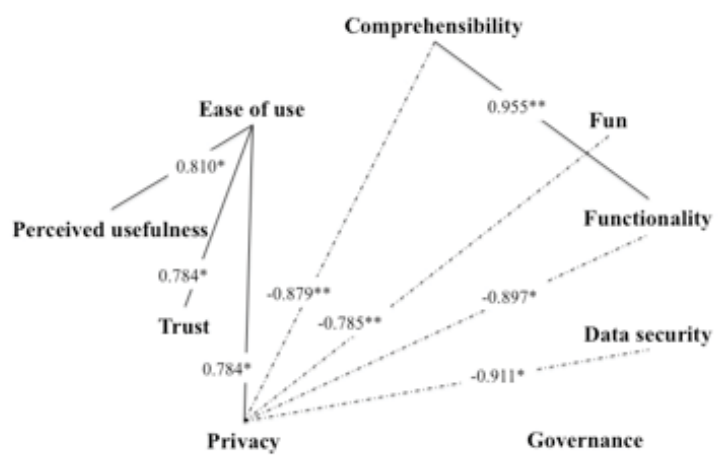

Intention to use

Figure 4. Interrelation between usability dimensions for the older user group

Ease of use and usefulness - the traditional usability measures are highly interrelated and ease of use shows high positive correlations to trust and privacy. Interestingly, though, the 
majority of measures are negatively related. The wish for privacy decreases with low comprehensibility, functionality, fun and data security. Neither aspect is related to the intention to use and to perceived governance when using telemedical systems.

\section{DISCUSSIONAND FUTURE RESEARCH}

In this study, an extension of the classical usability and technology acceptance approach was applied in a telemedical

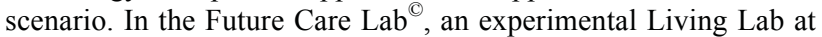
RWTH Aachen University, participants were requested to interact with a telemedical application and to evaluate the usability of the system. Beyond the traditional measures - the ease of using a system and perceived usefulness - we explored additional dimensions, such as the wish to protect the privacy, trust, functionality, and fun when interacting with the system, as well as governance, i.e. the perceived ability to control it. Overall, the system usability was evaluated positively: Most of the criteria under study yielded high evaluations in both age groups. However, results show that traditional measures fall short in novel application scenarios such as health supporting systems in home environment. For the younger group the traditional usability and technology acceptance criteria (ease of use and usefulness) do not relate to any of the other examined aspects, which show high interrelations among each other. In fact, users' wish for privacy protection, trust in technology, and fun in interaction with the system are major aspects that should be considered for a more comprehensive usability concept. For the older group, yet, the traditional acceptance measures are significant sources, which do relate to each other (a high usefulness evaluation is accompanied by a high ease of use). Interestingly though, the negative intercorrelations between other measures show in general a more reluctant acceptance pattern for older adults. Their usability rating is mostly associated with high sensibility to privacy violations.

It can be concluded that the traditional usability concept should be amended whenever technology is integrated into private home environments [10], because the usage context in which technology is applied decisively determines the usability and acceptance requirements [2] [16], and should be addressed in future usability approaches.

As limitations the comparably small sample size of the study should be noted. For a first exploratory analysis this seems to be sufficient to understand the basic potential of extending the traditional usability definition. However, whenever the approach might be further evolved to a stable usability measurement, a considerably larger sample size should be examined. Beyond the need for a larger sample size, also further user characteristics should be considered. In the eHealth context, the inclusion of ailing persons for a better understanding of their usability requirements is therefore essential. A further factor is the cultural sensitivity. In this research, only insights into a Western European culture were gained. However, it is conceivable that other cultural values and norms may be sensitive to the use of technology in home environments [3].

\section{ACKNOWLEDGEMENTS}

Authors thank Lars Klack, Kai Kasugai and Felix Heidrich for their valuable research input and their indispensable support in the Future Care $\mathrm{Lab}^{\circ}$. Thanks also to Marie Hommelsen and Julia van Heek for further research support.

\section{REFERENCES}

[1] Alagöz, F., Ziefle, M., Wilkowska, W. and Calero Valdez, A. 2011. Openness to accept medical technology - a cultural view. In Information Quality in eHealth, A. Holzinger, K.-M.
Simonic, eds. Lect Notes Comput Sc, 7058, Springer, Berlin, 51-170.

[2] Arning, K., Gaul, S. and Ziefle, M. 2010. Same Same but Different. How Service Contexts of Mobile Technologies Shape Usage Motives and Barriers. In HCI in Work \& Learning, Life \& Leisure, Leitner et al. eds. Lect Notes Comput Sc, 6389, Springer, Berlin, 34-54.

[3] Choon, Y.-Y. 2005. Cross-Cultural Issues in HumanComputer Interaction, In: International Encyclopedia of Ergonomics and Human Factors, W. Karwowski, Ed. Taylor \& Francis, London, 1063-1069.

[4] Davis, F. D., Bagozzi, R. P., and Warshaw, P. R. 1989. User acceptance of computer technology: a comparison of two theoretical models. Manage Sci., 35, 8, 982-1003.

[5] Gaul, S. and Ziefle, M. 2009. Smart Home Technologies: Insights into Generation-Specific Acceptance Motives. In HCI and Usability for e-Inclusion, A. Holzinger, K. Miesenberger, eds. Lect Notes Comput Sc, 5889, Springer, Berlin, 312-332.

[6] Hassenzahl, M. 2004. The Thing and I: Understanding the relationship between user and product. In Funology. From usability to enjoyment, M.A. Blyhte et al., eds. Kluwer, Norwell, M.A., 31-42.

[7] Hommelsen, M. 2010. Usability criteria for multitouch interfaces in ehealth. Unpublished Bachelor thesis, RWTH Aachen University, Germany.

[8] Klack, L., Schmitz-Rode, T., Wilkowska, W., Kasugai, K., Heidrich, F., \& Ziefle, M. 2011. Integrated Home Monitoring and Compliance Optimization for Patients with Mechanical Circulatory Support Devices. Ann Biomed Eng, 39, 12, 2911-2921.

[9] Kleinberger, T., Becker, M., Ras, E., Holzinger, A. and Muiller P. 2007. Ambient Intelligence in Assisted Living: Enable Elderly People to Handle Future Interfaces. Lect Notes Comput Sc, 4555, Springer, Berlin, 103-112.

[10] Lalou, S. 2008. Identity, Social Status, Privacy and Facekeeping in the Digital Society. Soc Sc Inform, 47, 3, 299-330.

[11] Leonhardt, S. 2006. Personal Healthcare Devices. In AmIware Hardware Technology Drivers of Ambient Intelligence Mekherjee, S., et al. eds., Springer, Dordrecht, 349-370.

[12] Seshamani, M and Gray, A. 2004. Time to death and expenditure: an improved model for the impact of demographic change on health care, Age Ageing, 33, 6, 556-561.

[13] Wilkowska, W. and Ziefle, M. 2011. User diversity as a challenge for the integration of medical technology into future home environments. In Human-Centred Design of eHealth Technologies. Concepts, Methods and Applications, M. Ziefle, C. Röcker, eds. IGI Global, Hershey, P.A, 95-126.

[14]Wilkowska, W. and Ziefle, M. 2012. Privacy and Data Security in E-health: Requirements from Users' Perspective. Health Informatics $J, 18,3,191-201$.

[15] Ziefle, M. and Jakobs, E.-M. 2010. New Challenges in Human Computer Interaction: Strategic Directions and Interdisciplinary Trends. 4th International Conference on Competitive Manufacturing Technologies. University Stellenbosch, South Africa, 389-398.

[16] Ziefle, M., Himmel, S. and Wilkowska, W. 2011. When your Living Space Knows what you do. In Information Quality in eHealth, A. Holzinger, K.-M. Simonic, eds. Lect Notes Comput Sc, 7058, Springer, Berlin, 607-624.

[17] Ziefle, M. and Schaar, A.K. 2011. Gender differences in acceptance and attitudes towards an invasive medical stent. Electron. J. Health Inform, 6, 2, 1-18. 
